\title{
Ênfases e lacunas nas contribuições da prospecção de futuro no design para inovação social
}

Emphasis and gaps in the contributions of future prospecting in design for social innovation

KRASSUSKI, Camila; Mestranda; Universidade Federal do Paraná

camilafdo@gmail.com

CHAVES, Liliane; Dra. Universidade Federal Fluminense

chaves.liliane@gmail.com

HEEMANN, Adriano; Dr. Universidade Federal do Paraná

adriano.heemann@gmail.com

\section{Resumo}

Cada vez mais o design para inovação social torna-se um assunto pertinente e importante quando se trata de futuro e busca por soluções. Para investigar ênfases e lacunas a respeito dos temas, a metodologia de Revisão Bibliográfica Sistemática (RBS) foi utilizada para buscar, selecionar, analisar e comparar publicações existentes sobre o tema e, após, foi feita uma Revisão Bibliográfica Assistemática (RBA) para complementar os estudos. Como resultado, 16 publicações foram selecionadas, analisadas integralmente e sintetizadas. Por meio de um quadro teórico comparativo e avaliação de critérios, foi possível perceber que existem oportunidades de pesquisa no campo da prospeç̧ão de futuro no design para inovação social, principalmente quanto à prospecção e suas ferramentas e a atuação do design no campo das inovações sociais.

Palavras Chave: inovação social, design, prospecção, futuro.

\begin{abstract}
Increasily, design for social innovation becomes an important and pertinent issue to be discussed, considering that future, and search for solutions have been on focus. To investigate lacks and opportunities in the area, methods of a systematic literature review, followed by an assistematic literature review complementary was applied to analyse, compair and synthetize publications. Using a theorical frame, and analysing criterias, it was possible to realize that are opportunities of researches in the fields of foresight studies in design for social innovation, specially concerned to foresight and tools and the design on social innovation field.
\end{abstract}

Keywords: social innovation, design, foresight, future. 


\section{Introdução}

Inseridos no contexto da vida líquida proposta por Bauman (2008) - segundo o qual nada é definitivo, tudo está em transição - os indivíduos, as empresas, as organizações e a sociedade estão se relacionando, vivendo, produzindo e consumindo de modo diferente. As inovações sociais surgem nessa realidade líquida que, intensificada por problemas socioambientais cada vez mais evidentes, demanda novas alternativas para solução de problemas e melhoria de vida de maneira sustentável.

Bas \& Guillo (2015) acreditam que com o novo paradigma social baseado no acesso global, nas mudanças constantes, no imediatismo e redes sociais, as organizações vêm buscando mais empatia com os usuários. Essa mudança implica na necessidade de encontrar formas mais flexíveis, participativas e criativas para se aproximar da pesquisa de prospecção. Prospecção (foresight) é um termo que "indica o reconhecimento da habilidade de se apropriar do futuro, emancipando-se das supostas leis naturais que podem predeterminar o caminho para esse futuro." (BAS \& GUILLO, 2015, pág. 273).

Os temas inovação e prospecção encontram-se interligados. Segundo Bas \& Guillo (2015), prospecção e inovação são muitas vezes consideradas "áreas de conhecimento" ou "disciplinas" distintas, mas podem ser vistas como duas dimensões diferentes da mesma coisa: a prospecção como metodologia transversal e a inovação como dimensão inerente ao pensamento estratégico e à gestão. De acordo com Westley \& Antadze (2010): Para que as instituições e os sistemas sociais, que são as inovações sociais, permaneçam resilientes, é necessário, uma integração contínua da novidade.

O termo inovação social apresenta na literatura uma vasta gama de definições. Para André e Abreu (2006) implica em iniciativas que escapam à ordem estabelecida, uma mudança social qualitativa, uma alternativa ou uma ruptura. Cloutier (2003) indica que a inovacão social, tem o objetivo de gerar bem estar aos indivíduos e às comunidades e envolve cooperação entre diversos atores que buscam resultados tangíveis ou intangíveis. Porém, embora ocorra uma expansão do conceito de inovação, a concentração dos estudos acadêmicos se dá na tradicional inovação tecnológica, voltada mais a processos e produtos (BIGNETTI, 2011). Apesar de explicações para o crescimento e difusão de inovações de mercado, existem limitações para a aplicação no contexto da inovação social (WESTLEY \& ANTADZE, 2010).

Por meio de um levantamento de publicações e autores que abordam os temas design para inovação social e prospeç̧ão de futuro e a partir da análise das publicações, o objetivo deste estudo é levantar as ênfases e eventuais lacunas nas contribuições da prospecção de futuro no design para inovação social. Para contribuir com tal propósito, este estudo caracteriza-se como uma revisão teórico-conceitual de síntese temática (DRESCH; LACERDA; ANTUNES JR., 2016). Como etapas de investigação, adotou-se como procedimentos metodológicos uma Revisão Bibliográfica Sistemática (RBS) seguida de uma Revisão Bibliográfica Assistemática (RBA). Os temas prospecção de futuro e design para inovação social delimitaram os filtros de busca.

Considerando a seleção de sete estudos por meio da RBS e outros nove escolhidos a partir da RBA, em sua totalidade, 16 materiais entre livros, artigos científicos, publicações e guias, foram lidos na íntegra e analisados, compondo a base bibliográfica do presente artigo. 
A partir da confecção de um quadro teórico avaliando critérios pré-determinados, as 16 publicações foram analisadas buscando levantar as ênfases e lacunas nas contribuições da prospecção de futuro no design para inovação social. Ao final da análise, notou-se que a conceituação de inovação social nos artigos selecionados é atendida em quase todas as publicações. Como lacuna, percebeu-se uma falta de especificidade quando trata-se de design para inovação social e conceituação de prospecção e aplicação de ferramentas para prospecção. Com isso, constatou-se que existem oportunidades de pesquisa no campo da prospecção de futuro no design para inovação social a serem realizadas.

\section{Método}

Este estudo caracteriza-se como uma revisão teórico-conceitual de síntese temática (DRESCH; LACERDA; ANTUNES JR., 2016). Como etapas de investigação, adotou-se como procedimentos metodológicos uma Revisão Bibliográfica Sistemática (RBS) e uma Revisão Bibliográfica Assistemática (RBA).

\subsection{Revisão Bibliográfica Sistemática (RBS)}

Como método para realizar a coleta de dados para estruturação do artigo, fez-se o uso da Revisão Bibliográfica Sistemática apresentada por Conforto, Amaral e da Silva (2011), também conhecida como RBS, propõe que a pesquisa na literatura, em periódicos e plataformas de busca seja rigorosa e estruturada. A revisão apresenta três fases: a Entrada, o Processamento e a Saída, e tal ordem não deve ser modificada, conforme Figura 1.

Figura 1 - Etapas de uma Revisão Bibliográfica Sistemática
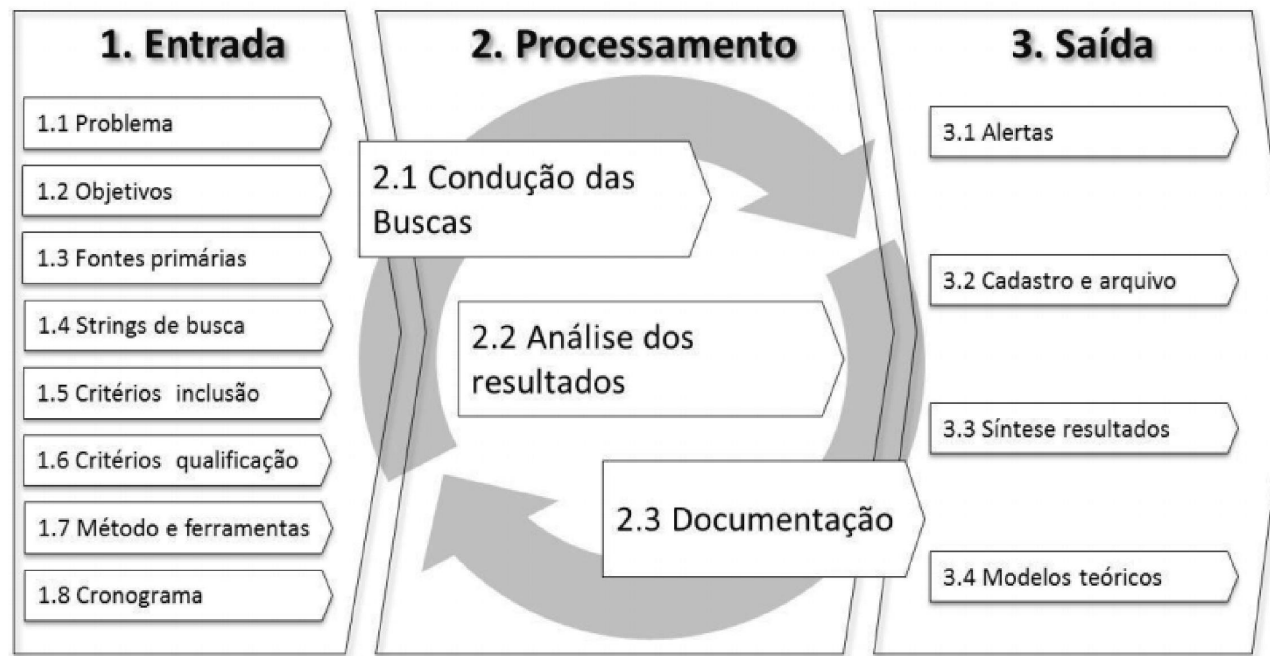

Fonte: Conforto, Amaral e da Silva (2011, p. 7)

A primeira etapa da RBS, a Entrada, iniciou-se com o problema de pesquisa, que neste caso é a identificação de publicações em periódicos nacionais e internacionais em que constem especificamente estudos de prospecção sobre o design para novação social. Portanto, a pergunta de pesquisa pode ser definida da seguinte forma: "Quais as ênfases e lacunas encontradas nos trabalhos publicados sobre as contribuições da prospecção de futuro no design para inovação 
social?".

Após a definição do problema, foi feita a delimitação dos objetivos, que nortearam a pesquisa, que para o presente trabalho é a apresentação de um estudo de avaliação de publicações em periódicos nacionais e internacionais que reúnam as áreas de estudo prospecção de futuro e design para inovação social. Com os objetivos pré-determinados, foram selecionadas as fontes primárias em que as publicações seriam pesquisadas. Quatro plataformas de busca foram escolhidas como fontes primárias de busca, sendo elas: BDTD, CAPES, Scopus e Scielo.

Ainda na Entrada, definiram-se as strings de busca que, de acordo com Conforto, Amaral e Silva (2011), são sequências de termos organizados utilizando operadores lógicos e combinando palavras referentes ao escopo de busca. De acordo com as principais temáticas de pesquisa, os termos encontrados no objetivo de pesquisa foram destrinchados e combinados para gerar as strings, assim como palavras correlatas. Primeiramente, foi estabelecido que apenas uma string seria na língua portuguesa e o restante seria na língua inglesa. A escolha foi feita pois os termos em inglês, tem a capacidade de abrangência de pesquisa muito mais ampla.

Nota-se a dificuldade em definir as strings para prospecção já que na literatura palavras como prediction, induction, trends, futurism, forecast apresentam definições próximas. A conclusão que se chega é que a palavra foresight (ou em português, prospecção) é o termo ideal para o qual o artigo se propõe a abordar pois foresight" implica na visão de um futuro múltiplo e com espaço para construção e pressupõe o encorajamento da pró-atividade e reconhecimento da habilidade de se apropriar do futuro." (BAS \& GUILLO, 2008, pág. 276).

Para o termo "prospecção", o objetivo era escolher palavras que auxiliassem a encontrar conteúdos afins a futuro, sucesso e previsão de futuro. Com isso, definiu-se que os seguintes palavras iriam compor as strings de busca: future (futuro), foresight,(prospecção), success (sucesso) e prediction (prognóstico). Na segunda temática, "design para inovação social" foi feita a separação em dois termos, obtendo-se "social innovation" e design. Com isso, as strings de busca foram definidas, conforme a Figura 2:

Figura 2 - Strings de busca utilizados

\begin{tabular}{|} 
"social innovation" and "design" and "future" \\
"social innovation" and "design" and "foresight" \\
"social innovation" and "design" and "success" \\
"social innovation" and "prediction" \\
"inovação social" and "design" \\
"social innovation" and "design"
\end{tabular}

Fonte: Os autores (2017)

Considerando os objetivos e o problema de pesquisa definidos, foi possível escolher os critérios de inclusão dos artigos. Optou-se por buscar nas quatro plataformas selecionadas, primeiramente utilizando filtros automáticos, sem qualquer limitação de data ou período pesquisado, apenas incluindo artigos revisados por pares e no idioma inglês. Após, foi feita a inclusão de uma filtragem e por meio do próprio mecanismo dos buscadores, foi possível excluir os estudos que não correlacionavam as temáticas com propostas como: healthy, economics, 
climate change, architecture, entre outros. Finalizando a fase de Entrada, as etapas do processo foram feitas de acordo com o cronograma preestabelecido, entre os meses de abril e maio de 2017.

Dando início à fase de Processamento, as buscas foram conduzidas, reduzindo a artigos não repetidos e selecionados de acordo com a avaliação e leitura de seu título, palavras-chave e resumo pertinentes ao escopo, diminuindo de forma significativa o número de artigos selecionados. Após, foi feita uma segunda filtragem através da leitura das introduções e conclusões dos estudos; ao final, a leitura completa dos artigos selecionados. Na última etapa do Processamento, realizou-se a documentação do procedimento, identificando strings, plataformas e artigos encontrados inicialmente e a quantidade restante após passar pelas filtragens. A efeito de documentação e organização, foram realizados os arquivamentos dos artigos selecionados em pastas virtuais no computador pessoal. E, por fim, elaborou-se a síntese de resultados.

\subsection{Revisão Bibliográfica Assistemática (RBA)}

A RBA foi realizada com o intuito de adquirir fontes de informação de forma assistemática, ou seja, sem que houvesse uma operação metódica e organizada para a sua busca de fontes. Dessa forma, livros, artigos e outros materiais que possuem relevância reconhecida para o escopo do presente estudo foram escolhidos (materiais físicos ou digitais). Tais materiais foram selecionados por meio de indicação de profissionais e professores ou mesmo encontrados mas referências bibliográficas de alguns materiais filtrados na RBS. A etapa da RBA foi feita após a conclusão da RBS. A próxima seção aponta os resultados alcançados em ambas as etapas de pesquisa (sistemática e assistemática), assim como os critérios adotados nos mecanismos de busca.

\section{Resultados}

\subsection{Resultados da Revisão Bibliográfica Sistemática}

Considerando as quatro bases de dados, a partir da primeira busca em fontes primárias, foi encontrado um total de 231 artigos, sendo: 17 da base BDTD (por ser uma plataforma brasileira, foi utilizada apenas uma string com palavras em português), 115 da CAPES, 95 da Scopus e quatro da Scielo, como mostra a Tabela 1:

Tabela 1: Trabalhos encontrados por plataforma a partir da busca de fontes primárias

\begin{tabular}{|c|c|c|c|c|}
\hline String & BDTD & CAPES & Scopus & Scielo \\
\hline "social innovation" and "design" and "future" & - & 46 & 49 & 1 \\
\hline "social innovation" and "design" and "foresight" & - & 6 & 4 & 0 \\
\hline "social innovation" and "design" and "success" & - & 20 & 21 & 0 \\
\hline "social innovation" and "prediction" & - & 4 & 6 & 0 \\
\hline "inovação social" and "design" & 17 & 33 & 0 & 0 \\
\hline "social innovation" and "design"* & - & 6 & 15 & 3 \\
\hline TOTAL POR BASE & 17 & 115 & 95 & 4 \\
\hline TOTAL & \multicolumn{4}{|c|}{231} \\
\hline
\end{tabular}

Fonte: os autores (2017) 
Após a busca primária, foram excluídos artigos duplicados. Na etapa seguinte, com a leitura de título, palavras-chave e resumo, foram selecionados 21 trabalhos que mais atendiam ao escopo da pesquisa proposta. A partir desses artigos, foram feitas as leituras de suas introduções e conclusões, resultando na escolha de sete que foram lidos na íntegra. A Tabela 3 apresenta os estudos resultantes dos processos de busca por nome de autor em ordem alfabética:

Tabela 3: Estudos selecionados por meio de RBS

\begin{tabular}{|c|c|c|c|c|c|c|c|c|}
\hline & Autor & Título & Palavras-chave & Tipo & Idioma & País & Ano & Base \\
\hline 1 & $\begin{array}{l}\text { BAS, E., \& } \\
\text { GUILLO, M. }\end{array}$ & $\begin{array}{l}\text { Participatory foresight } \\
\text { for social innovation. } \\
\text { FLUX-3D method (For- } \\
\text { ward Looking User } \\
\text { Experience), a tool for } \\
\text { evaluating innovations. }\end{array}$ & $\begin{array}{l}\text { Participatory foresight } \\
\text { Users' experience } \\
\text { Innovation culture } \\
\text { Design thinking Change } \\
\text { management }\end{array}$ & Artigo & Inglês & Espanha & 2013 & Scopus \\
\hline 2 & $\begin{array}{l}\text { FARRINGTON, } \\
\text { T.; HENSON, } \\
\text { K.; CREWS, C. }\end{array}$ & $\begin{array}{l}\text { The Use of Strategic } \\
\text { Foresight Methods for } \\
\text { Ideation and Portfolio } \\
\text { Management. }\end{array}$ & $\begin{array}{c}\text { Inductive } \\
\text { scenarios, Technology } \\
\text { vforecasting, Foresight } \\
\text { vtools, Portfolio } \\
\text { management }\end{array}$ & Artigo & Inglês & $\begin{array}{l}\text { Estados } \\
\text { Unidos }\end{array}$ & 2012 & CAPES \\
\hline 3 & GUILLO, M & $\begin{array}{c}\text { Futures, } \\
\text { communication and } \\
\text { social innovation: using } \\
\text { participatory foresight } \\
\text { and social media } \\
\text { platforms as tools for } \\
\text { evaluating images of } \\
\text { the future among } \\
\text { young people }\end{array}$ & $\begin{array}{l}\text { Images of the future, } \\
\text { Open innovation, } \\
\text { Participatory foresight, } \\
\text { Social innovation, } \\
\text { Creativity, Social media }\end{array}$ & Artigo & Inglês & Espanha & 2013 & CAPES \\
\hline 4 & $\begin{array}{l}\text { MANZINI, E.; } \\
\text { RITHAA, M. K. }\end{array}$ & $\begin{array}{l}\text { Distributed Systems } \\
\text { And Cosmopolitan } \\
\text { Localism: An Emerging } \\
\text { Design Scenario For } \\
\text { Resilient Societies. }\end{array}$ & $\begin{array}{c}\text { Resilience, } \\
\text { Development, } \\
\text { Distributed systems, } \\
\text { Social innovation, Design }\end{array}$ & Artigo & Inglês & $\begin{array}{l}\text { Itália e } \\
\text { África } \\
\text { do Sul }\end{array}$ & 2016 & Scopus \\
\hline 5 & $\begin{array}{l}\text { MORAIS-DA- } \\
\text { SILVA, R. L.; } \\
\text { TAKAHASHI, A. } \\
\text { R. W.; } \\
\text { SEGATTO, A. P }\end{array}$ & $\begin{array}{c}\text { Scaling up social } \\
\text { innovation: a meta- } \\
\text { synthesis. }\end{array}$ & $\begin{array}{c}\text { Social innovation, } \\
\text { Scalability, Enlargement. } \\
\text { Meta-synthesis, Creating } \\
\text { social value. }\end{array}$ & Artigo & Inglês & Brasil & 2016 & CAPES \\
\hline 6 & $\begin{array}{l}\text { MORJARIA, N; } \\
\text { ROSS, \& MAY, } \\
\text { A. }\end{array}$ & $\begin{array}{c}\text { Fostering social } \\
\text { innovation: identifying } \\
\text { lead users for } \\
\text { participatory design. }\end{array}$ & $\begin{array}{l}\text { Human Factors, } \\
\text { Participatory Design, } \\
\text { Lead User Method }\end{array}$ & Artigo & Inglês & $\begin{array}{l}\text { Reino } \\
\text { Unido }\end{array}$ & 2013 & CAPES \\
\hline 7 & $\begin{array}{l}\text { VOLTAN, A.; } \\
\text { DE FUENTES, } \\
\text { C. }\end{array}$ & $\begin{array}{l}\text { Managing multiple } \\
\text { logics in partnerships } \\
\text { for scaling social } \\
\text { innovation }\end{array}$ & $\begin{array}{c}\text { Institutional logics, } \\
\text { Partnership, Social } \\
\text { innovation, Scaling social } \\
\text { innovation }\end{array}$ & Artigo & Inglês & Canadá & 2016 & CAPES \\
\hline
\end{tabular}

Fonte: Os autores (2017) 
Os estudos selecionados foram lidos na íntegra e analisados, compondo a base bibliográfica do presente artigo.

\subsubsection{Síntese das publicações (RBS)}

Após a execução de todas as etapas da RBS, foram selecionados sete artigos que estavam em sintonia com o escopo de pesquisa delimitado. Tais artigos foram lidos em sua íntegra e sintetizados.

Bas e Guillo (2015) discorrem acerca da conceituação atual e histórica do termo Foresight como um dos pressupostos para entender a inovação. Para eles, diferentemente da previsão, a prospecção tem uma abordagem positivista que depende de atitudes proativas para acessar o "futuro". Os autores propõem no artigo, "sete chaves" para alcançar a inovação em organizações e acreditam que o Fluxo 3D (que prevê feedbacks sobre a satisfação do usuário, assim como dimensões, indicações e variáveis) é a ferramenta ideal para ajudar na tomada de decisões a curto, médio e longo prazo em organizações. Por meio da demonstração de estudos de caso, concluem que a ferramenta Fluxo 3D é uma forma de prospecção participativa, que se encaixa de forma adequada no cenário em que a sociedade está inserida.

O artigo elaborado por Crews, Farrington e Henson (2012) relata a experiência do uso estratégico de métodos de prospecção para ideação e desenvolvimento de novos produtos para a empresa PepsiCo. Alguns colaboradores participaram do workshop, baseando-se em cenários em um futuro de cinco a 10 anos. $O$ artigo relata que o processo auxiliou a empresa a identificar possíveis mercados do futuro e a gerenciar o programa de inovação de longa duração para a empresa. Consideraram essencial o engajamento da equipe no objetivo da dinâmica.

Guillo (2013) aborda em seu artigo resultados de um estudo comparativo sobre imagens do futuro encontradas entre jovens estudantes espanhóis e finlandeses. Por meio de um "workshop do futuro" e uma survey, o autor incita que os estudantes pensem no futuro, analisando valores culturais e mensagens que podem influenciar a maneira como o futuro é pensado, desejado e esperado.

Considerando o dinamismo da sociedade contemporânea Manzini e Rithaa (2016), observam um novo mundo emergente, com pessoas buscando sistemas distribuídos, rápidos e flexíveis. Assim, desenvolvem as noções de sistemas distribuídos, sistemas resilientes, localismo cosmopolita, discutindo a possibilidade do uso de ferramentas do design para conectá-los e reiterá-los. Segundo os autores, sistemas resilientes não poderiam existir sem inovação social, que, por sua vez, não poderiam ser reais sem que houvesse uma comunidade criativa por trás, composta por empreendedores criativos interessados em inventar e melhorar soluções para o dia a dia apenas recombinando e reconfigurando fatores já existentes.

Motivados pela lacuna encontrada na área de pesquisa em inovação social sobre a escalabilidade ou capacidade de replicar um modelos de negócio ou serviço das organizações, o foco principal de Takahashi et. al (2016) é por meio da metodologia de pesquisa exploratórioindutiva composta por oito passos, proposta por Hoon (2013), avaliar quais são os fatores responsáveis por promover a escalabilidade de uma inovação social. Analisando sistematicamente 66 artigos, a síntese do estudo apontou que os fatores que propiciam a escalabilidade de uma 
inovação social podem ser internos, dependendo de aspectos como o empreendedor e a organização, como também podem ser externos. O estudo buscou contribuir auxiliando os gestores de inovações sociais na etapa da ampliação da atuação da organização.

O foco da pesquisa para de Marjoria, Ross e May (2013) foi o de identificar usuários líderes (pessoas que utilizam de forma assídua o produto ou serviço) para o design participativo, fomentando a inovação social. O destaque do artigo está centrado na importância da identificação e recrutamento do usuário líder, assim como suas necessidades, demonstrando o estudo por meio de um caso de transporte sustentável. Segundo os autores, a importância da integração de usuários líderes em processos participativos é uma maneira de aumentar potencialmente a capacidade de inovação dos resultados, uma vez que esse usuário considera em primeira ordem as necessidades e desejos futuros. Coletar estudos sobre as tendências também se faz importante na metodologia indicada pelo estudo.

Voltan e De Fuentes (2016) apresentam um estudo exploratório sobre como inovadores sociais gerenciam lógicas concorrentes no contexto de parcerias para escalabilidade de uma inovação social. Utilizam como metodologia um estudo de caso para analisar o sucesso na escalabilidade de inovações sociais. No trabalho, apresentam uma estrutura teórica, incluindo o papel do aprendizado organizacional e teorias cognitivas no contexto da inovação social e empresas sociais, focando especificamente em aproximações colaborativas entre organizações que frequentemente desenvolvem esforços na escalabilidade da inovação social.

\subsection{Resultados da Revisão Bibliográfica Assistemática}

A Tabela 4 apresenta os estudos resultantes dos processos de busca por nome de autor em ordem alfabética:

Tabela 4: Estudos selecionados por meio de RBA

\begin{tabular}{|c|c|c|c|c|c|c|}
\hline & Autor & Título & Tipo & Idioma & País & Ano \\
\hline 1 & ANDRÉ, I.; ABREU, A & Dimensões e espaços da inovação social & Artigo & Português & Portugal & 2006 \\
\hline 2 & BIGNETTI, L.P & $\begin{array}{l}\text { As inovações sociais: uma incursão por ideias, } \\
\text { tendências e focos de pesquisa }\end{array}$ & Artigo & Português & Brasil & 2011 \\
\hline 3 & BISHOP, P.; HINES, A & $\begin{array}{c}\text { Thinking About the Future; Guidelines for } \\
\text { Strategic Foresight }\end{array}$ & Livro & Inglês & $\begin{array}{l}\text { Estados } \\
\text { Unidos }\end{array}$ & 2015 \\
\hline 4 & $\begin{array}{l}\text { BROWN, T., \& } \\
\text { WYATT, J. }\end{array}$ & Design Thinking for Social Innovation & Artigo & Inglês & $\begin{array}{l}\text { Estados } \\
\text { Unidos }\end{array}$ & 2010 \\
\hline 5 & CLOUTIER, J. & Qu'est-ce que l'innovation sociale? & Artigo & Francês & Canadá & 2003 \\
\hline 6 & MANZINI, E. & $\begin{array}{c}\text { Design para inovação social e sustentabilidade: } \\
\text { comunidades criativas, organizações } \\
\text { colaborativas e novas redes projetuais }\end{array}$ & Livro & Português & Itália & 2008 \\
\hline 7 & $\begin{array}{l}\text { MULGAN,G; ALI,R.; } \\
\text { HALKETT, R.; } \\
\text { SANDERS,B. }\end{array}$ & $\begin{array}{l}\text { In and Out of Sync: The Challenge of Growing } \\
\text { Social Innovations }\end{array}$ & Guia & Inglês & $\begin{array}{l}\text { Reino } \\
\text { Unido }\end{array}$ & 2007 \\
\hline 8 & $\begin{array}{l}\text { MURRAY, R.; } \\
\text { CAULIER-GRICE, J.; } \\
\text { MULGAN, J. }\end{array}$ & The Open Book of Social Innovation & Livro & Inglês & $\begin{array}{l}\text { Reino } \\
\text { Unido }\end{array}$ & 2010 \\
\hline 9 & $\begin{array}{l}\text { WESTLEY, F., \& } \\
\text { ANTADZE, N. }\end{array}$ & $\begin{array}{l}\text { Strategies for scaling social innovation for } \\
\text { greater impact. }\end{array}$ & Artigo & Inglês & Canadá & 2010 \\
\hline
\end{tabular}

Fonte: os autores (2017) 
Considerando a RBA um complemento importante de pesquisa, foram selecionados mais nove estudos que auxiliaram na conceituação de termos e contribuíram para uma visão mais ampla das temáticas abordadas neste artigo.

\subsubsection{Síntese das publicações (RBA)}

André e Abreu (2006) discutem o conceito de inovação social e identificam suas principais dimensões analíticas. Para isso, constroem uma matriz para sistematizar e comparar os estudos de caso sobre o papel da inovação social no desenvolvimento de territórios.

Bignetti (2011) demonstra em seu estudos as diferenças existentes entre inovações sociais e inovações tecnológicas e, com isso, sugere a necessidade de gestões e condução de modelos distintos para cada uma das inovações. Além disso, analisa a evolução histórica do termo inovação social por meio de três focos: indivíduos, organizações e movimentos.

Bishop e Hines (2015) apresentam informações que guiam a prospeç̧ão estratégica. Organizados em seis grupos (enquadramento, scanning, previsão, visão, plano e ação, demonstram aspectos que se fazem essenciais para a compreensão da atividade de prospecção estratégica.

Brown e Wyatt (2010), demonstram em seu estudo a origem do design thinking e os espaços de atuação da metodologia. Entendem que assim como pode ser utilizado para melhorar produtos e serviços, o design thinking vem sendo cada vez mais aplicado por designers para desenvolver melhores soluções para inovações sociais. Os autores também abordam cases em que o design thinking auxiliou na proposição de soluções para questões sociais.

O objetivo de Cloutier (2003), é apresentar as definições do tema inovação social por diversos pesquisadores e comparar as semelhanças e diferenças entre seu prórprio estudo. A linha de pesquisa de Cloutier é feita a partir de três linhas complementares: território, condições de vida e trabalho e emprego.

Manzini (2008) concentra-se em atender as inovações sociais no senso da comunidade e destaca a questão ambiental e o empenho por modos de vida mais sustentáveis. O autor propõe plataformas habilitantes como ferramentas para mudanças e foca na contribuição que a inovação social pode trazer ao tema design para a sustentabilidade em termos de design estratégico e design de serviços.

Mulgan, Rushanara, Halkett e Sanders (2007) concentram-se em abordar como as inovações sociais crescem e se difundem. Baseando-se em 11 estudos de casos de inovação social, os autores elaboram um guia fundamentado em termos teóricos e empíricos para pessoas envolvidas na inovação social como inovadores, investidores e políticos, destacando as condições necessárias para promover o crescimento ou escalabilidade das inovações.

Murray, Caulier-Grice e Mulgan (2010) em "Open book for social innovation" demonstram formas de projetar, desenvolver e promover o crescimento das inovações sociais. Por meio de descrições e exemplificações de métodos e ferramentas, os autores fornecem um passo a passo para como desenvolver uma inovação social em diversos meios. 
Westley e Antadze (2010) em seu estudo, exploram estratégias e dinâmicas para escalar inovações sociais. Os autores propõem um modelo distinto de transformação do sistema de grupos de inovações sociais que dependem de mudanças descontínuas e de escala cruzada. Conceituam o termo sistema social, que contribui para o entendimento de crescimento de uma inovação social.

A sessão seguinte discute o conteúdos dos estudos selecionados, traçando um panorama sobre as ênfases e lacunas nas contribuições da prospecção no design para inovação social.

\section{Discussão}

Após a leitura e análise dos 16 estudos selecionados, apresenta-se um quadro teórico composto pelos autores levantados e critérios estabelecidos que buscam identificar ênfases e lacunas nas contribuições da prospecção no design para inovação social. Compondo este quadro, cada trabalho selecionado recebeu um conceito de zero a dois, sendo que o conceito 0 não atende ao critério em análise; o conceito 1 atende parcialmente e o conceito 2 atende com profundidade. Os critérios analisados foram elaborados com o intuito de encontrar as ênfases e eventuais lacunas nas temáticas prospecção e design para inovação social, sendo eles:

a) Aborda/ conceitua inovação social? - Importância do critério para avaliar os autores que se aprofundaram em um dos principais temas do presente estudo;

b) Aborda/ conceitua design para inovação social? - Critério relevante pois delimita os estudos que abordam especificamente sobre o design para inovação social, ou sejam os autores que se aprofundam nos estudos sobre projetar inovação social;

c) Aborda/ conceitua prospecção/ foresight? - Outra temática importante a ser avaliada, mais um dos conceitos principais abordados no estudo;

d) Apresenta ferramentas para prospecção/ foresight? - Este critério é importante pois a prospecção é realizada com auxílio de ferramentas;

e) Contribui com previsões ou cenários sobre futuro? - Futuro foi uma das palavras que contribui para a definição das strings de busca nas revisões bibliográficas;

f) Aborda/ conceitua escalabilidade para inovações sociais? - O conceito de escalabilidade emergiu espontaneamente durante as leituras, e percebeu-se que é de extrema importância para avaliar o futuro das inovações sociais, pois comtempla crescimento e difusão;

g) Contribui com a temática prospecção no design para inovação social? - O último critério é a resposta a pergunta de pesquisa proposta neste artigo. 
Tabela 5: Quadro teórico

\begin{tabular}{|c|c|c|c|c|c|c|c|c|c|c|c|c|c|c|c|c|}
\hline \multirow[b]{2}{*}{ CRITÉRIOS | AUTORES } & \multicolumn{7}{|c|}{ RBS } & \multicolumn{9}{|c|}{ RBA } \\
\hline & 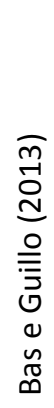 & 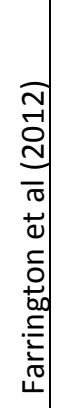 & 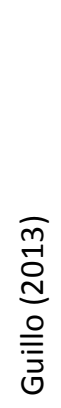 & 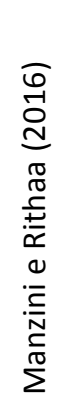 & 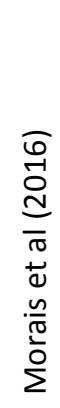 & 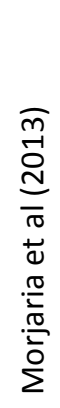 & 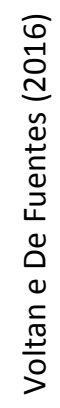 & 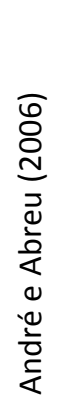 & 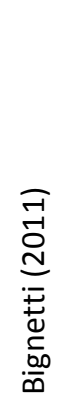 & 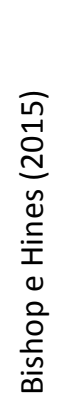 & 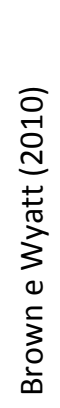 & 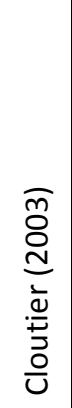 & 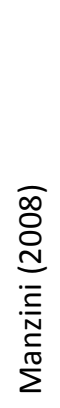 & 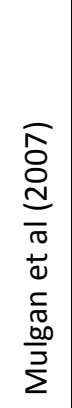 & 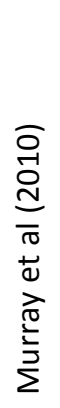 & 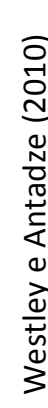 \\
\hline $\begin{array}{l}\text { a) Aborda/ conceitua } \\
\text { inovação social? }\end{array}$ & 1 & 0 & 1 & 2 & 2 & 0 & 2 & 2 & 2 & 0 & 0 & 2 & 2 & 2 & 2 & 2 \\
\hline $\begin{array}{l}\text { b) Aborda/ conceitua design } \\
\text { para inovação social? }\end{array}$ & 2 & 0 & 0 & 2 & 1 & 1 & 0 & 0 & 0 & 0 & 2 & 0 & 2 & 2 & 2 & 1 \\
\hline $\begin{array}{l}\text { c) Aborda/ conceitua } \\
\text { prospecção/ foresight? }\end{array}$ & 2 & 2 & 1 & 0 & 0 & 1 & 0 & 0 & 0 & 2 & 0 & 0 & 0 & 0 & 0 & 0 \\
\hline $\begin{array}{l}\text { d) Apresenta ferramentas } \\
\text { para prospecção/ foresight? }\end{array}$ & 2 & 2 & 1 & 0 & 0 & 2 & 0 & 0 & 0 & 2 & 0 & 0 & 0 & 0 & 0 & 0 \\
\hline $\begin{array}{l}\text { e) Contribui com previsões } \\
\text { ou cenários sobre futuro? }\end{array}$ & 2 & 2 & 2 & 1 & 0 & 2 & 1 & 0 & 0 & 2 & 1 & 0 & 0 & 1 & 1 & 0 \\
\hline $\begin{array}{lr}\text { f) Aborda/ } & \text { conceitua } \\
\text { escalabilidade } & \text { para } \\
\text { inovações sociais? } & \\
\end{array}$ & 0 & 0 & 0 & 0 & 2 & 0 & 2 & 0 & 0 & 2 & 0 & 0 & 2 & 2 & 2 & 2 \\
\hline $\begin{array}{l}\text { g) Contribui com a temática } \\
\text { prospecção no design para } \\
\text { inovação social? }\end{array}$ & 2 & 0 & 0 & 0 & 0 & 1 & 0 & 0 & 0 & 0 & 0 & 0 & 0 & 1 & 0 & 0 \\
\hline CONCEITO FINAL & 11 & 6 & 5 & 5 & 5 & 7 & 5 & 2 & 2 & 6 & 3 & 2 & 4 & 8 & 7 & 5 \\
\hline
\end{tabular}

Fonte: Os autores (2017)

Ao final do quadro, no campo "conceito final", é possível identificar a pontuação total de cada autor e com isso, selecionar os autores que possuíram maior relevância ao tema.

Nota-se que Bas e Guillo (2013), em seu estudo "Participatory foresight for social innovation. FLUX-3D method (Forward Looking User Experience), a tool for evaluating innovations)", são os autores que mais abordam todos os critérios analisados, seguido por Mulgan et al (2007) com o artigo "In and Out of Sync: The Challenge of Growing Social Innovations". Em terceiro lugar, Morjaria et al (2013) e Murray et al (2010), com seus respectivos estudos "Fostering social innovation: identifying lead users for participatory design" e "The Open Book of Social Innovation".

Buscando responder às ênfases encontradas nas contribuições da prospecção de futuro no design para inovação social, percebe-se que a maioria dos autores conceitua de forma satisfatória a inovação social, porém, alguns não estão centrados na questão do "design para inovação social", pois há uma diferença entre eles: quando inclui-se a palavra design, a questão projetual e a 
competência do designer são evidenciados, caso contrário, a inovação social é apenas conceituada e trabalhada em outros aspectos que não os de projetar.

Por meio do levantamento dos estudos, encontrou-se algumas ênfases nas contribuições da prospecção de futuro no design para inovação social. Menos da metade dos estudo selecionados apresentou ferramentas de prospecção, o que indica uma lacuna a ser atendida. Quanto ao critério da abordagem do termo prospecção ou foresight, considerando esta revisão bibliográfica, apenas três deles o conceitua com profundidade, ou seja, falta uma especificação em relação a esse assunto quando trata-se design para inovação social. Com a aplicação dos conceitos, foi possível perceber que apenas três dos 16 estudos analisados contribui com a temática prospeç̧ão no design para inovação social, ou seja, existem oportunidades de pesquisa nesse campo a serem realizadas.

\section{Conclusão}

Oriundas da realidade líquida (BAUMAN, 2008) e intensificadas por problemas socioambientais, as inovações sociais buscam a resolução de problemas e melhoria de vida de maneira sustentável. Por sua vez, a prospecção de futuro é a metodologia inerente ao pensamento estratégico e à gestão (BAS \& GUILLO, 2015). Por meio de revisões bibliográficas, buscou-se as ênfases e eventuais lacunas nas contribuições da prospecção de futuro no design para inovação social.

Caracterizado por ser uma revisão teórico-conceitual de síntese temática (DRESCH.; LACERDA; ANTUNES JR, 2016) e utilizando os métodos de revisão bibliográfica sistemática e assistemática, o objetivo do estudo foi o de levantar as ênfases e eventuais lacunas nas contribuições da prospecção de futuro no design para inovação social. Após a análise na íntegra das publicações selecionadas, foi possível perceber que existem oportunidades de pesquisa no campo da prospecção de futuro no design para inovação social a serem realizadas. Quanto à apresentação de ferramentas de prospecção, poucos autores trataram do assunto, assim como a conceituação do termo prospeç̧ão, o que indica um campo a ser aprofundado.

Como recomendação para futuros trabalhos, indica-se abordar de forma mais aprofundada a conceituação do design para a inovação social, contribuindo para a realização das competências do designer nesse campo de atuação. Percebe-se que muitas vezes, as inovações sociais são tratadas pela ótica da sociologia ou estudos pertinentes à área administrativa. Porém, existe um grande potencial de atuação do designer na aplicação de ferramentas de prospecção para contribuir no campo da implantação, desenvolvimento, sustentação, crescimento ou difusão das inovações sociais. 


\section{REFERÊNCIAS}

ANDRÉ, I.; ABREU, A. Dimensões e espaços da inovação social. Finisterra, XLI, 81, 2006, pp. 121141

BAUMAN, Z. Modernidade líquida. Rio de Janeiro: Zahar, 2000.

BIGNETTI, L.P. As inovações sociais: uma incursão por ideias, tendências e focos de pesquisa. Ciências Sociais Unisinos 47(1):3-14, 2011

BISHOP, P.; HINES, A. Thinking About the Future; Guidelines for Strategic Foresight. Social Technologies, Houston, 2007.

BAS, E., \& GUILLO, M. Participatory foresight for social innovation. FLUX-3D method (For- ward Looking User Experience), a tool for evaluating innovations. Technological Forecasting and Social Change, 101(December), 275-290, 2015.

BROWN, T., \& WYATT, J. Design Thinking for Social Innovation. Stanford Social Innovation Review, Stanford, 2010.

CLOUTIER, J. Qu'est-ce que l'innovation sociale? Cahier du CRISES Collection Études théoriques no ET0314 SBN : 2-89605-135-X Dépôt légal : Québec, 2003.

CONFORTO, E. C.; AMARAL, D. C.; DA SILVA, S. L. Roteiro para revisão bibliográfica sistemática: aplicação no desenvolvimento de produtos e gerenciamento de projetos. $8^{\circ}$ Congresso Brasileiro de Gestão de Desenvolvimento de Produto, Porto Alegre, 2011.

DRESH A.; LACERDA D. P.; ANTUNES J. A. V. Design Science Research: Método de Pesquisa para Avanço da Ciência e Tecnologia. Porto Alegre: Bookman, 2015.

FARRINGTON, T.; HENSON, K.; CREWS, C. The Use of Strategic Foresight Methods for Ideation and Portfolio Management. Research-Tchnology Management, 2012.

GUILLO, M. Futures, communication and social innovation: using participatory foresight and social media platforms as tools for evaluating images of the future among young people. Published online - Springerlink.com, 2013.

MANZINI, E. Design para inovação social e sustentabilidade: comunidades criativas, organizações colaborativas e novas redes projetuais. Rio de Janeiro: E-Papers, 2008.

MANZINI, E.; RITHAA, M. K. Distributed Systems And Cosmopolitan Localism: An Emerging Design Scenario For Resilient Societies. Sustainable Development, Wiley Online Library, 2016.

MORAIS-DA-SILVA, R. L.; TAKAHASHI, A. R. W.; SEGATTO, A. P. Scaling up social innovation: $a$ meta-synthesis. RAM, Rev. Adm. Mackenzie [online]. 2016, vol.17, n.6, pp.134-163, 2016.

MORJARIA, N; ROSS, \& MAY, A. Fostering social innovation: identifying lead users for participatory design. CHItaly '13, September 16 -20 2013, Trento, Italy, 2013.

MULGAN, G., ALI. R., HALKETT, R.; SANDERS, B. In and Out of Sync: The Challenge of Growing Social Innovations: London: NESTA, 2007.

MURRAY, R.; CAULIER-GRICE, J.; MULGAN, J. The Open Book of Social Innovation, NESTA 


\section{Artigo Completo}

Innovating Public Services: London, 2010.

VOLTAN, A. ; DE FUENTES, C. Managing multiple logics in partnerships for scaling social innovation, European Journal of Innovation Management, (2016) Vol. 19 Issue: 4, pp.446-467, https:// doi.org/10.1108/EJIM-01-2016-0010

WESTLEY, F., \& ANTADZE, N. Making a difference: strategies for scaling social innovation for greater impact. Innovation Journal, 15(2), 2-18, 2010. 